\title{
APPLICATIONS OF ANALYSIS TO THE ARITHMETIC OF HIGHER FORMS*
}

\author{
BY
}

E. T. BELL

1. The classical applications of elliptic functions to the theory of numbers are confined to a limited range of problems concerning enumerative functions in a narrow strip of additive arithmetic. The historically first instance is typical of all. In it there is determined more readily by means of elliptic functions than by any other method the number of representations of an integer as a sum of four squares. Class number formulas have essentially the same characteristics: the range is everywhere bounded by enumerative theorems on representations in homogeneous forms of the second degree.

2. As a preliminary extension it was shownt that the range can be broadened to include paraphrases involving $L$-functions of any parity. In this extension the associated forms are still of the second degree. The simplest cases of the paraphrases thus obtained are enumerative theorems of the kind just described. For if $f(x)$ is of parity $p(1 \mid 0)$ we can take as a very special case $f(x)=1$ for all values of $x$.

3. The wider range in $\S 2$ can again be extended indefinitely in a new direction. It will be shown that we can always find general relations (generalized paraphrases) concerning $L$-functions of any parity and of any order in which the arguments of the $L$-functions are linear functions of the indeterminates which represent an arbitrary constant integer in forms of degree $n_{1}$ and order $n_{1}^{\prime}$ with at least one of the coefficients in the forms an arbitrary constant integer, where $n_{1}>n, n_{1}^{\prime}>n^{\prime}$, and $n, n^{\prime}$ are any preassigned integers $>0$. We pass therefore from the preliminary paraphrases of $\S 2$ in which the associated forms are of the second degree to like paraphrases in which the forms are of any degree.

4. The extended paraphrases of $\S 3$ can be yet further generalized. We shall show that it is sufficient in forming the arguments of the $L$-functions to include only those values of the indeterminates which are such that arbitrary constant even positive powers of them lie within any preassigned limits.

\footnotetext{
* Presented to the Society, San Francisco Section, October 21, 1922.

† These Transactions, vol. 22 (1921), p. 1. We presuppose $\S \S 1,2,5,6,8,9$ of that paper.
} 
5 . It will be shown that the extension in $\S 4$ includes as a special case that in $\S 3$, which in turn includes the extension in $\S 2$, and this again includes the type of result outlined in $\S 1$. Although we have referred above only to elliptic functions it will be evident (cf. $\S 6$ of the former paper) that a precisely similar analysis applies to relations between the multiple theta functions and that the presence of periodic quotients in such relations is not a prerequisite for the applications of the method.

To preserve the continuity of the discussion we omit all illustrations, a detailed set of which will be given in another paper.

6 . Let $\lambda, \mu, \cdots, \nu$ denote arbitrary odd constant integers $>0$, and $a, b, \cdots, c$ arbitrary constant integers $\neq 0$. Let

$$
\varsigma_{\alpha p}(w)=1, \quad(\alpha, p, w) \equiv(\lambda, a, x), \quad(\mu, b, y), \cdots,(\nu, c, z)
$$

if $w$ is of the form $p t^{\alpha}$ where $t$ is an integer $\gtreqless 0$, and in all other cases let $\varphi_{\alpha p}(w)=0$. Since $\alpha$ is odd it is evident that $\varphi_{\alpha p}(w)$ is an even function of $w$. It is also clear that the similarly defined function $\varphi_{\beta p}(w)$ in which $\beta$ is not odd is neither an even nor an odd function of $w$. Let $f \equiv f(x, y, \cdots, z)$ have in addition to the property

(A) $f$ is single valued whenever its arguments $x, y, \cdots, z$ are simultaneously integers $\gtreqless 0$;

one (and necessarily only one) of the following properties:

(B) $f$ is an even function of each of its arguments;

$(C) f$ is an odd function of each of its arguments;

$\left(B^{\prime}\right) f$ is an even function of all of its arguments,

$$
f(x, y, \cdots, z)=f(-x,-\cdot y, \cdots,-z)
$$

$\left(C^{\prime}\right) f$ is an odd function of all of its arguments,

$$
f(x, y, \cdots, z)=-f(-x,-y, \cdots,-z) .
$$

Beyond the specific pair of these properties which $f$ possesses it is wholly arbitrary. The last is essential in what follows.

If now $f$ has the property $A$ and any one $A^{\prime}$ of $B, C, B^{\prime}, C^{\prime}$, so also has

$$
\varphi_{\lambda a}(x) \varphi_{\mu b}(y) \cdots \varphi_{\nu c}(z) f\left(\sqrt[\lambda]{\frac{x}{a}}, \sqrt[\mu]{\frac{y}{b}}, \cdots, \sqrt[\nu]{\frac{z}{c}}\right),
$$

considered as a function of the arguments $x, y, \cdots, z$, the same pair $A, A^{\prime}$ of properties. 
7. Consider all the sets $(x, y, \cdots, z)(i=1,2, \cdots)$ of integers satisfying a given set $S$ of conditions. Among these some (or none) will also satisfy the conditions

$$
x_{i}=a \xi_{i}^{\lambda}, \quad y_{i}=b \eta_{i}^{\mu}, \quad \cdots, \quad z_{i}=c \zeta_{i}^{\nu},
$$

where the only restriction upon $\xi_{i}, \eta_{i}, \cdots, \zeta_{i}$ is that they shall be integers $\gtreqless 0$. Let $\left(\xi_{i}, \eta_{i}, \cdots, \zeta_{i}\right)(i=1,2, \cdots)$ be all the sets of values of the $\xi_{i}$, $\eta_{i}, \cdots, \zeta_{i}$ obtained in this way.

Suppose we have found the relation

$$
\sum_{i} k_{i} f\left(x_{i}, y_{i}, \cdots, z_{i}\right)=0
$$

in which the $k_{i}$ are numerical constants, the sum extends to all sets $\left(x_{i}, y_{i}, \cdots, z_{i}\right)$, and $f$ has the properties $A, A^{\prime}$. Since $f$ is unrestricted beyond $A, A^{\prime},(2)$ implies

$$
\sum_{i} k_{i} \varphi_{\lambda a}\left(x_{i}\right) \varphi_{\mu b}\left(y_{i}\right) \cdots \varphi_{\nu c}\left(z_{i}\right) f\left(\sqrt[\lambda]{\frac{x_{i}}{a}}, \sqrt[\mu]{\frac{y_{i}}{b}}, \cdots, V^{\nu} \overline{\frac{z_{i}}{c}}\right)=0,
$$

which by (1) and the definition of $\varphi$ is equivalent to

$$
\sum_{i} k_{i} f\left(\xi_{i}, \eta_{i}, \cdots, \zeta_{i}\right)=0
$$

the sum extending to all sets $\left(\xi_{i}, \eta_{i}, \cdots, \zeta_{i}\right)$. Only these sets appearing in (3), we may use (1) to replace the set $S$ of conditions upon the $\left(x_{i}, y_{i}, \cdots, z_{i}\right)$ by a set $S^{\prime}$ upon the $\left(\xi_{i}, \eta_{i}, \cdots, \zeta_{i}\right)$. We may then consider (3) directly in relation to $S^{\prime}$ without the intermediary $S$. If (2) is a paraphrase of the kind cited in $\S 2$ for $L$-functions of one or other of the parities $p(1,1, \cdots, 1 \mid 0)$, $p(0 \mid 1,1, \cdots, 1), p(n \mid 0), p(0 \mid n)$, where $n$ is the number of independent variables in the set $(x, y, \cdots, z)$ and the unit in the first two symbols is repeated $n$ times, then (3) is a result of the sort described in $\S 3$ for the same $L$-functions.

8. To reach the further extension summarized in $\S 4$ we now proceed as follows. We are to select only the $\left(\xi_{i}, \eta_{i}, \cdots, \zeta_{i}\right)$ in the limited region

$$
r \leqq \xi_{i}^{g} \leqq \varrho, \quad s \leqq \eta_{i}^{h} \leqq \sigma, \quad \cdots, \quad t \leqq \zeta_{i}^{k} \leqq \tau
$$

where $r, \varrho, s, \sigma, \cdots, t, \tau$ are arbitrary constants (but consistent with the respective inequalities) and $g, h, \cdots, k$ are arbitrary constant even integers $>0$. 
Let the function $\theta_{p j \pi}(x)=1$ if $p \leqq x^{j} \leqq \pi$, where $p, \pi$ are numerical constants, and in all other cases let this function vanish. Then if $j$ is an even integer $>0, \theta_{p j \pi}(x)$ is an even function of $x$, while if $j$ is not an even integer, the function is neither even nor odd.

Hence, by an argument precisely similar to that in $\S 7$, if $\left(\xi_{i}^{\prime}, \eta_{i}^{\prime}, \cdots, \zeta_{i}^{\prime}\right)$ $(i=1,2, \cdots)$ are all the sets $\left(\xi_{i}, \eta_{i}, \cdots, \zeta_{i}\right)$ which satisfy in addition to $S^{\prime}$ the conditions (4), we may replace (3) by

$$
\sum_{i} k_{i} f\left(\dddot{\xi}_{i}^{\prime}, \eta_{i}^{\prime}, \cdots, \zeta_{i}^{\prime}\right)=0
$$

Finally then we combine (4) and $S^{\prime}$ into one set $S^{\prime \prime}$ of conditions, and as in $\S 7$ consider (5) directly in relation to $S^{\prime \prime}$. The result is as described in $\S 4$ for $L$-functions of the parities indicated in $\$ 7$.

9. For the special values $(r, g, \varrho),(s, h, \sigma), \cdots,(t, k, \tau)=(0,2, \infty)(5)$ becomes (3), and when further in (1) we put

$$
(a, \lambda)=(b, \mu), \cdots,(c, \nu)=(1,1),
$$

(3) degenerates to (2). Hence (5) includes both (3), (2).

10. We say that $S^{\prime \prime}$ has been obtained from $S$ by extension. In extending a given set of conditions upon a particular system of indeterminates we therefore subject the system to the further conditions (1), (4). Now a paraphrase of the preliminary type (former paper, p. 5) refers to a separation in which no indeterminate occurs to a power higher than the second. By extension as above we replace such a separation by an extended set of conditions in which:

(i) Some (or all) of the indeterminates occur to arbitrary odd constant powers which may have the same or different exponents;

(ii) The coefficients of the terms involving these powers are arbitrary constant integers;

(iii) The ranges of variation of the indeterminates in (i) are so restricted that an arbitrary constant even power of each indeterminate lies within any preassigned limits.

The result of this extension is called an extended separation. As shown above this extension includes the kind of the former paper as one of its simplest cases.

11. If $n$ is as in $\S 7$ the number of independent variables in the set $(x, y, \cdots, z)$ the function $f$ in (2), (3), (5) is, by $B, C, B^{\prime}, C^{\prime}$ of $\S 6$, of one or other of the parities indicated in $\S 7$. The generalization of all results thus far obtained follows immediately upon repeated application of the 
argument in $\S \S 7,8,9$, and we can pass at once from a paraphrase of the form (2) in which $f$ is now an $L$-function of parity

$$
p\left(a_{1}, a_{2}, \cdots, a_{r} \mid b_{1}, b_{2}, \cdots, b_{s}\right)
$$

where the $a$ 's and $b$ 's are any integers $>0$, to equations (3) and (5) in which now $f$ is of the same parity and the summation in (5) is performed with respect to an extended separation. In this, $f$ retains its complete generality beyond the specific equations of parity which it satisfies.

In the special case previously discussed we considered $L$-functions subject to restrictions beyond their parity conditions. The restrictions were chiefly of the type that the function changes sign or remains invariant under permutation of some or all of its variables. Clearly all such results can be carried over to the extended case.

12. The extended principle of paraphrase can now be stated.

Any identity in elliptic, abelian or theta functions, provided only that it contains the arguments of the functions and is not merely an identity betiveen constants, implies and is implied by an identity between general $L$-functions integrated over an extended separation.

UNIVERSITY OF WASHINGTON, Seattle, Wash. 